\title{
Determination of the Braking Period Duration in Case of an Electric Motor Driving Mechanism with a Hydrodynamic Coupling
}

\author{
Atila ZELIĆ, Rastislav ŠOSTAKOV, Dragan ŽIVANIĆ
}

\begin{abstract}
Hydrodynamic coupling is a power-transmitting device with no stiff kinematic relation between the shafts of its basic parts (pump and turbine impellers) and no torque transformation. It has been used in various driving mechanisms due to its certain advantages, especially in transient working regimes of machines. Nevertheless, no sufficient attention has been devoted to the study of a braking period of such driving mechanisms, either in research papers or in practice. The existing few papers analyse only the experimental results concerning the braking period. The paper outlines a combined graphical and numerical method which enables the survey a of driving system operation in either steady-state or transient working regimes. The method is based on mechanical characteristics of all relevant driving system components defined either experimentally or in manufacturers' catalogues. The method is explained through defining the braking period duration on an example of a typical belt conveyor driving mechanism with a hydrodynamic coupling. The importance of the proposed method for the practice, and certain limitations of its application are pointed out.
\end{abstract}

Keywords: braking period duration; electric motor driving system; hydrodynamic coupling; transient operating regime

\section{INTRODUCTION}

The application range of hydrodynamic power transmission has been considerably extended since the first half of the 20th century. In spite of that, a lot of presentday engineers are not sufficiently familiar with operation details of a complete machine containing a hydrodynamic component. Therefore, machine designers often adopt other concepts of electric motor driving systems (EDS), even when the solution with a hydrodynamic component would be better in every way. Therefore, any new research that thoroughly considers the relevant operating regimes of hydrodynamic components within the EDS, can be of a valuable help in finding the solution of practical problems.

Despite the efficiency and wide spectrum of technical potentials of contemporary frequency controlled electric motors, hydrodynamic power transmission is still present in driving mechanisms of transporting, earthmoving, mining and power plant machines.

Hydrodynamic coupling (HC) as a component of an EDS is still used due to its advantages:

- Simple structure and high operating reliability, combined with relatively low maintenance demands,

- Low mass and volume per transmitted power unit,

- Enables short run-up and fast pass through the adverse operating regime of an electric motor (EM), followed by the slow run-up of a working device (WD), and lower inertial forces (especially important for setting heavy large mobile machines in motion),

- Possibility of a drive run-up along with exceptionally high resistance forces acting on a WD (during a heavy loaded belt conveyor restart after some hitch),

- Protection of an EM against overloading,

- Automatic adaptation of a HC to the variations of the working resistance (WR), etc.

This list of advantages leads to a conclusion that the main advantages by use of $\mathrm{HC}$ emerge during the transient operating regimes of an EDS.

In the majority of research papers dealing with the operating regimes of a $\mathrm{HC}$ (see e.g. $[1,2,4])$, above all, the run-up of an EDS has been analysed. In spite of that, braking regimes of an EDS and behaviour of a $\mathrm{HC}$ during these processes have been only negligibly studied, in both professional and scientific papers. Some of few available researches are presented in $[9,10]$, but the $\mathrm{HC}$ braking regime was analysed only partially, based on simulation models. That is important for the optimisation of existing simulation models, estimate of the relevancy of parameters, and the accuracy of calculations, etc. But, that demands the experimental verification which is rarely possible in the phase of machine design. In such case parameters of transient regime can be defined with the needed accuracy using the catalogue data of relevant machine components.

Therefore, the intention of the paper is to try to:

- Remove some dilemmas regarding mutual connections of variables relevant for the analysis of EDS behaviour during transient operating regimes, and

- Present one appropriate procedure for an approximate estimate (but accurate enough for a practical usage) of the braking period duration of an EDS with a HC.

In accordance with the previous, the importance of a procedure for surveying the transient operating regimes of an EDS in a joint diagram is explained. Such access is based on the previous research results of the authors of the paper [2]. After that, a proposal for the combined graphical and numerical (GM) method for determining the braking period duration of an EDS with a mechanical brake (MB) and $\mathrm{aC}$ is put forward in the main part of the paper.

\section{TOPICS OF THE STUDY AND STARTING REMARKS}

During machine operation, periods occur when the values of variables of interest (parameters of mechanical power: force and velocity, i.e. torque and angular velocity) remain unchanged. Steady state conditions imply that the driving mechanism either is moving with a constant speed or is at a standstill. But, during transient operating periods the values of variables of interest vary over time.

In the paper, the method for a clear graphical outlining and easy-to-understand surveying of the main variables during transient operating regimes of an EDS is explained on an example of a typical belt conveyor drive mechanism (Fig. 1). It is well known that a $\mathrm{HC}$ is an often used component in such power transmission systems, [5], due to its already mentioned advantages. 
An analysis of belt conveyor run-up is one of the main topics of contemporary research in the field of continuous material transport (see e.g. $[6,7,8,11]$ ), especially in studies connected with reduction of energy expenses, belt life-time extension, etc. The harmful dynamic impacts are nowadays offen reduced by using expensive solutions, as frequency and voltage controlled EMs, PLC devices, etc, [12]. However, significant reduction of dynamic forces acting on the belt (often the most expensive component of a conveyor) can be achieved in a considerably cheaper way, by installing a $\mathrm{HC}$ into the high-power EDS.

If the controlled stopping of a belt conveyor is required (e.g. for a conveyor with inclined sections), a MB (drum or disc type) can be installed between an EM and a gear box (GB). Reliable data on the required braking period duration are necessary for the calculation of the braking torque needed for the belt stopping, i.e. for the selection of the corresponding MB parameters. In addition to that, data on the braking torque are also needed for the thermal proof of brake linings, for the estimate of the additional forces in the belt due to the deceleration, etc.

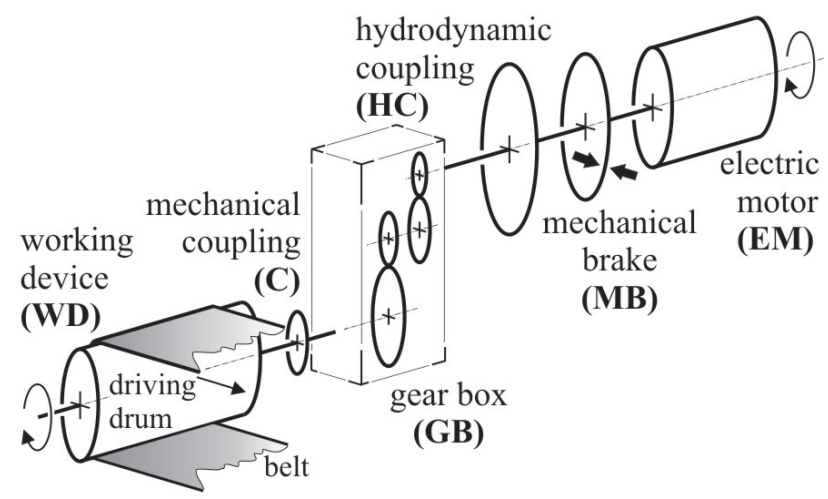

Figure 1 Schematic outline of EDS with $\mathrm{HC}$ and $\mathrm{MB}$ (example: belt conveyor)

Momentary state of the EDS shown in Fig. 1 - where "state" denotes the values of all the EDS variables of interest - represents the result of the electric motor (EM), power transmission (PT - includes $\mathrm{MB}, \mathrm{HC}, \mathrm{GB}$ and $\mathrm{C}$ ), and working device (WD) concurrence. Consequently, during the considered (in this case transient) operating period, the state of all EDS components has to be known, because their operating regimes are determined by the conditions of dynamic equilibrium.

In further consideration, the EDS shown in Fig. 1 is represented by its simplified mechanical model (Fig. 2) with two rotating masses, denoted as $J_{1}$ and $J_{2}$ (in fact equivalent mass moments of inertia), equivalent to all the masses on the pump impeller side and on the turbine impeller side. The connection between them realizes the $\mathrm{HC}$ torque $T_{H C}$. The $\omega_{1}$ and $\omega_{2}$ are the angular velocities of the pump and turbine impellers. Influence of the velocity ratio through the GB is taken into account in the procedure of reducing the values of variables. Masses of all moving components of the EDS (including the working fluid in a $\mathrm{HC}$ ) and torques/forces acting on them, are represented by the equivalent mass moments of inertia and torques. Their values are recalculated onto the HC shafts, using the well-known procedure based on the energy conservation law. The reduced values of EM, $\mathrm{MB}, \mathrm{HC}$ and WD variables obtained in this way, can be directly added, compared and represented in a diagram torque vs. angular velocity. a)

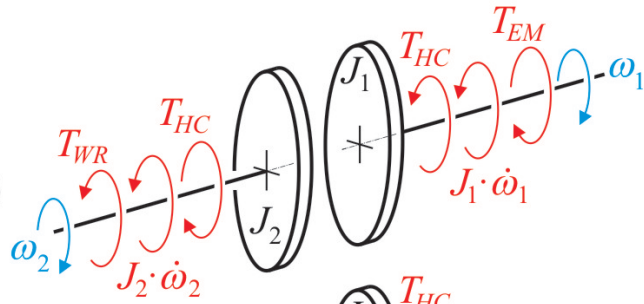

b)

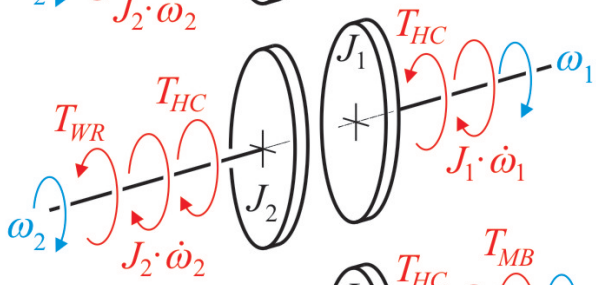

c)
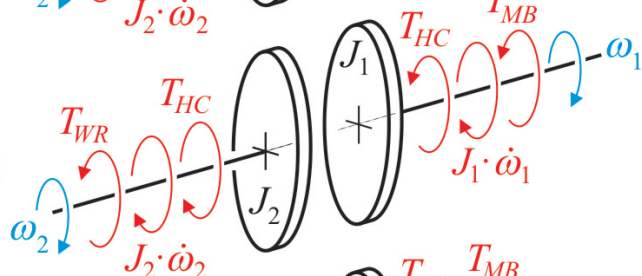

d)

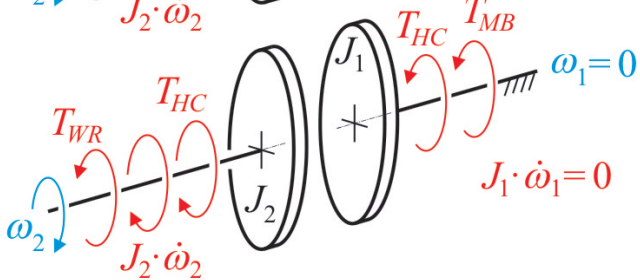

Figure 2 Mechanical models of EDS in Fig. 1: model of the run-up phase (a) and models of the braking phases B1 (b), B2 (c) and B3 (d)

In the paper, all further considerations are carried out on the assumption that all EDS components are adequately chosen. In aim to make the procedure of determining the braking period duration easy-to-understand, for the analysis of transient operating regimes an EDS is adopted, consisting of:

- A.C. squirrel-cage induction electric motor (EM),

- HC with constant fill and straight impeller blades (as the most economical variant of a $\mathrm{HC}$ for the adopted driving mechanism of a belt conveyor with inclined sections) and a slightly simplified and polished form of $\mathrm{HC}$ characteristic curves, in the diagram $T-i_{\omega}$ or $T-\omega_{2}$ with $\omega_{1}$ as the parameter (in aim to make them easierto-survey),

- $\quad$ MB with a constant braking torque $T_{M B}$, installed on the EM shaft,

- WD with a constant resistance torque $T_{W R}$, and

- Additional mechanical device - ratchet (e.g. one-way clutch with rollers - not shown in Fig. 1), that after the stopping of the HC pump impeller prevents the uncontrolled motion of the belt and the output part of the driving mechanism connected with the turbine impeller in the direction opposite to the direction of belt motion during normal operation, and

- Elastic and damping characteristics of all mechanical components are neglected.

However, previous simplifications do not influence either the general applicability of the presented method of EDS operation survey in a joint diagram, or the proposed procedure for the estimate of the braking period duration.

In aim to make easier the comprehension of the proposed method, graphical survey of the complete EDS operation in a single joint diagram is briefly explained in the next heading. 


\section{SURVEY OF EDS TRANSIENT OPERATING REGIMES IN A SINGLE JOINT DIAGRAM}

Generally speaking, operating process of an EM, PT and WD is determined by a series of mutually connected influences, e.g. mechanical, electric, magnetic, thermal, etc. These connections are expressed mostly in analytical or graphic form (using functions obtained by calculations or simulations, or on the basis of the experimental research results). In an analysis of operation of an EDS with a $\mathrm{HC}$, graphical description of steady state mechanical characteristics (lines defined by pairs of values of torque $T$ and angular velocity $\omega$ ) in a single diagram has certain advantages over an analytical form (e.g. better observing and easier comprehension of EDS operation even in the designing phase). This advantage is even more important in the education of future mechanical engineers.

Usually used methods for presenting the steady state characteristics of EM, MB, HC, and WD in diagrams $T-\omega$ are explained in details in $[2,3]$. It is important to stress that changes of EDS state (e.g. during transient operating regimes) cannot be exactly observed only based on these steady state characteristics. Besides, some variables necessary for the analysis of EDS operation, are only approximately defined, or obtained by testing the EDS components one by one, under certain conditions. In spite of all that, qualitative survey of EDS operation on the basis of steady state characteristics is successfully applicable in engineering practice, even for the approximate defining of the transient operating regime duration.

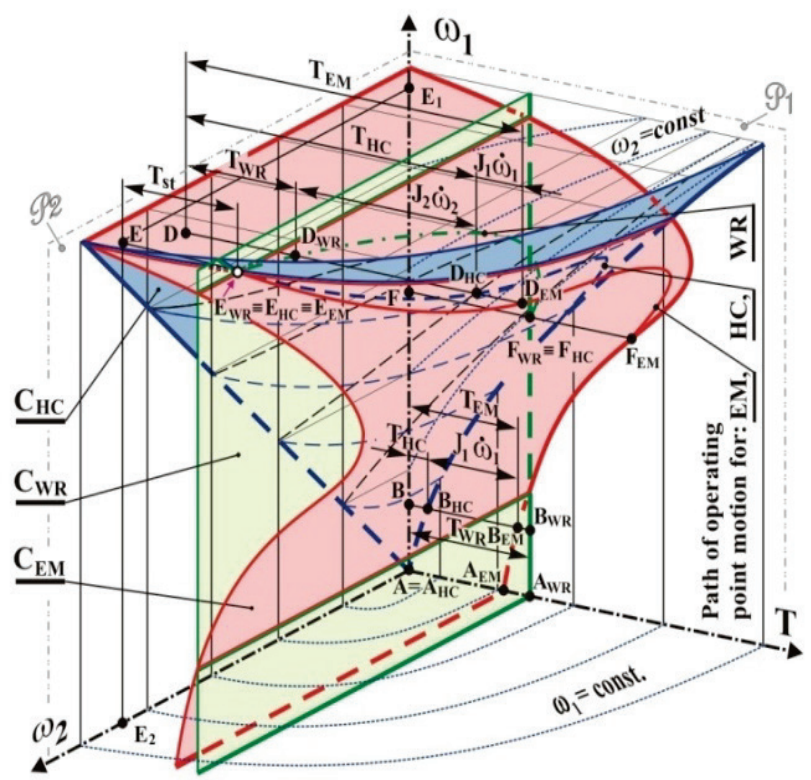

Figure 3 Graphical outline of EDS run-up process in the joint 3D diagram with mechanical characteristics $\mathrm{C}_{\mathrm{EM}}, \mathrm{C}_{\mathrm{HC}}$ and $\mathrm{C}_{\mathrm{WR}}$

As an illustration, in Fig. 3 is graphically represented the run-up of the EDS shown in Fig. 1. In the example of the EDS with no stiff kinematic relation (described by the model in Fig. 2.a), it is necessary to survey 3 parameters $\left(T, \omega_{1}\right.$, and $\left.\omega_{2}\right)$ at the same time in a 3-dimensional (3D) joint diagram. In aim to simplify the graphical outline, only the characteristics relevant for the driving regime of EM and the tractive regime of $\mathrm{HC}$ are shown. In this period the $\mathrm{MB}$ remains inactive and its characteristic is not in sight.
Accompanying explanations in the text are based on the procedure proposed by the author in [2].

Torque characteristic of a $\mathrm{HC}$ is defined by the torque value $T_{H C}$, dependent on the angular velocities $\omega_{1}$ and $\omega_{2}$. Therefore, it is represented as a spatially curved surface $\mathrm{C}_{\mathrm{HC}}$. Mechanical characteristic of an EM is independent of $\omega_{2}$, and represented as a cylindrically curved surface $\mathrm{C}_{\mathrm{EM}}$ perpendicular to the plane $\mathrm{P} 1$ defined by the axes $\omega_{1}$ and $T$. The resistance characteristic WD is independent of $\omega_{1}$, and represented as a plane $\mathrm{C}_{\mathrm{WR}}$ parallel to the plane $\mathrm{P} 2$ defined by the axes $\omega_{1}$ and $\omega_{2}$. The state of an EDS at an arbitrary moment is represented by the momentary position of the operating points (defined by the pairs of corresponding values $T$ and $\omega$ ) on these characteristics. All the operation points are on the same straight line parallel to the $T$-axis.

At the moment of switch-on, the operation point of EM starts from the point $\mathrm{A}_{\mathrm{EM}}$ moving along the intersection line of surface $\mathrm{C}_{\mathrm{EM}}$ and plane $\mathrm{P} 1$, while operating points of $\mathrm{HC}$ and WD start from points $\mathrm{A}_{\mathrm{HC}}$ and $\mathrm{A}_{\mathrm{WR}}$. During the motion, when the operating point of $\mathrm{EM}$ reaches the point $\mathrm{B}_{\mathrm{EM}}$, the part $J_{2}$ stands still due to $T_{H C}<T_{W R}\left(\omega_{2}=0\right)$. The difference $T_{E M}-T_{H C}$ (corresponds to the length $\mathrm{B}_{\mathrm{HC}} \mathrm{B}_{\mathrm{EM}}$ ) accelerates the part $J_{1}$, and $\omega_{1}$ is increasing. In the point $\mathrm{F}$ is $T_{H C}=T_{W R}$ $\left(\omega_{2}=0\right)$, i.e. operating points of $\mathrm{HC}$ and WD coincide with each other in point $F_{\mathrm{WR}} \equiv \mathrm{F}_{\mathrm{HC}}$. After that, the part 2 of EDS starts moving $\left(\omega_{2}>0\right)$, the operating point of EM leaves the plane P1, moving along the line $\mathrm{F}_{\mathrm{EM}} \mathrm{E}_{\mathrm{EM}}$ on the surface $\mathrm{C}_{\mathrm{EM}}$. The torque $T_{E M}$ (corresponds to the length $\mathrm{DD}_{\mathrm{EM}}$ ) overcomes the torque $T_{H C}\left(\mathrm{DD}_{\mathrm{HC}}\right)$, and the difference $T_{E M}$ $-T_{H C}\left(\mathrm{D}_{\mathrm{HC}} \mathrm{D}_{\mathrm{EM}}\right)$ goes on accelerating the part $J_{1}$. The line $\mathrm{F}_{\mathrm{HC}} \mathrm{E}_{\mathrm{HC}}$ on the surface $\mathrm{C}_{\mathrm{HC}}$ describes the motion of the operating point $\mathrm{HC}$. The torque $T_{H C}$ overcomes the torque $T_{W R}$ (corresponds to the length $\mathrm{DD}_{\mathrm{WR}}$ ), and the difference $T_{H C}-T_{W R}\left(\mathrm{D}_{\mathrm{WR}} \mathrm{D}_{\mathrm{HC}}\right)$ accelerates the part $J_{2}$. The motion of the operating points stops in the point $\mathrm{E}_{\mathrm{WR}} \equiv \mathrm{E}_{\mathrm{HC}} \equiv \mathrm{E}_{\mathrm{EM}}$, i.e. in the steady state position of operating points. This point is the joint intersection point of all three characteristics, i.e. surfaces $\mathrm{C}_{\mathrm{EM}}, \mathrm{C}_{\mathrm{HC}}$ and $\mathrm{C}_{\mathrm{WR}}$. Values of torques $T_{E M}=T_{H C}=T_{W R}$ are equal, and steady state angular velocities of pump and turbine impellers are $\omega_{1 s t}=$ const. (length $\mathrm{AE}_{1}$ ) $>\omega_{2 s t}=$ const. (length $\mathrm{AE}_{2}$ ).

\section{DETERMINING THE BRAKING PERIOD DURATION}

The braking process can be analysed in an analogous way to the previously explained procedure of surveying the EDS run-up regime. However, in this case it is necessary to add into the joint diagram the characteristic of $\mathrm{MB}$ $\left(\mathrm{C}_{\mathrm{MB}}\right)$, represented as a plane parallel to the plane P2, Fig. 4. In the process of stopping the HC pump impeller, the $\mathrm{HC}$ moves over to the overtaking regime $\left(\omega_{2}>\omega_{1}\right)$. Therefore, it is necessary to survey the motion of the operating points in the adjacent octant of the diagram, where the characteristic $\mathrm{C}_{\mathrm{MB}}$ is now of interest, too.

3D diagrams shown in Fig. 3 and 4 enable an insight into the operating regimes of EM, PT and WD in the same time. Regrettably, they are unsuitable for the determination of transient operation phases duration. In aim to complete this task successfully, it is suitable to move over into the 2D diagram, obtained by projecting all the characteristics onto the $T$ - $\omega_{1}$ plane, with $\omega_{2}$ as a parameter. 
The braking process for the adopted EDS consists of 3 braking phases and the total braking duration is determined as the sum of durations of these phases:

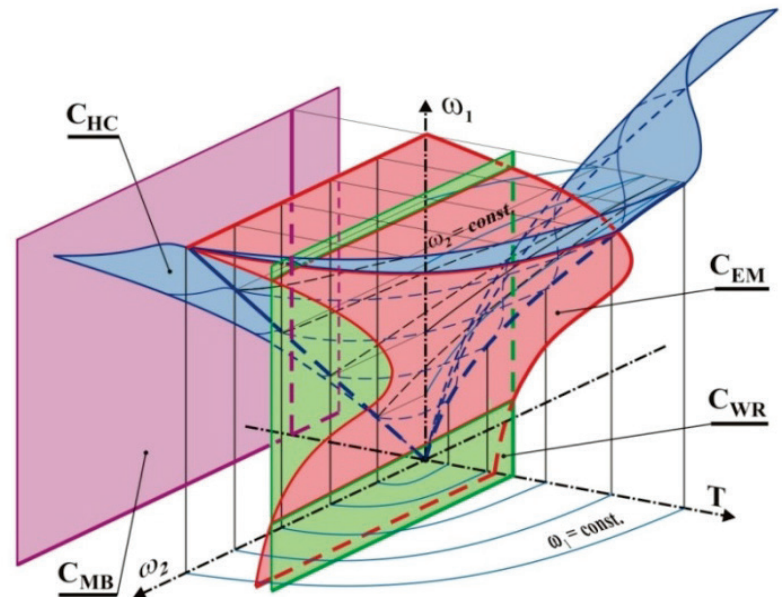

Figure 4 Graphic outline of mechanical characteristics $\mathrm{C}_{\mathrm{EM}}, \mathrm{C}_{\mathrm{MB}}, \mathrm{C}_{\mathrm{HC}}$ and $\mathrm{C}_{\mathrm{WR}}$ in the joint 3D diagram

1) Phase B1 (described by the model in Fig. 2.b) begins at the moment of EM power-supply cut-off. Therefore, the value of EM torque during all 3 phases is $T_{E M}=0$ ). The MB is activated at the same moment, but, due to the mechanical braking process based on the friction between the brake linings and its rotating component, braking does not start momentarily, but only after a short time period $t_{M B d}$ (brake reaction time). During this period the braking torque value is $T_{M B}=0$, but due to the working resistance, the whole EDS slows down, i.e. masses $J_{1}$ and $J_{2}$ decelerate (in general, at different rates).

2) Phase B2 (described by the model in Fig. 2.c) begins with the mechanical braking, and ends at the moment of the HC pump impeller coming to a standstill $\left(\omega_{1}=\right.$ $0)$. At the end of this phase the turbine impeller still rotates with the angular velocity $\omega_{2} \neq 0$.

3) Phase B3 (described by the model in Fig. 2.d) takes place by the already blocked HC pump impeller shaft, and during this phase the $\mathrm{HC}$ turbine impeller continues to decelerate until it comes to a standstill, too, $\left(\omega_{2}=0\right)$.

The proposed procedure for determining the braking period duration demands the usual $2 \mathrm{D}$ diagram of the HC mechanical characteristics family, with $\bar{\omega}_{1}$ as a parameter (Fig. 5.a). Characteristics are outlined in their „complete“ form, i.e. extended over 3 quadrants. It is suitable to use the relative coordinates: the transmission number $i_{\omega}$ on the $x$ axis, and the relative value of the torque $\bar{T}$ on the $y$-axis. Expressions of $i_{\omega}, \bar{\omega}_{1}, \bar{\omega}_{2}$, and $\bar{T}$ are defined in Fig. 5.a. It is suitable to adopt $\omega_{1 \max }=\omega_{s}$, where $\omega_{s}$ denotes the maximal value of EM angular velocity in the quadrant I (i.e. the EM synchron angular velocity). Adopting the rated EM torque value $T_{N}$ for the basis of the $\bar{T}$ value is not suitable due to its dependence on the EM duty class.

In aim to display all the characteristics $\left(\mathrm{C}_{\mathrm{EM}}, \mathrm{C}_{\mathrm{HD}}, \mathrm{C}_{\mathrm{MB}}\right.$ and $\mathrm{C}_{\mathrm{WR}}$ ) in a joint $2 \mathrm{D}$ diagram, before all, the family of curves (representing the HC tractive and overtaking operating regimes) in Fig. 5.a has to be transformed into a more suitable form, with $\bar{\omega}_{1}$ on the $x$-axis, $\bar{T}$ on the $y$-axis, and $\bar{\omega}_{2}$ as a parameter, Fig. 5.b. After that, the axes of $\bar{\omega}_{1}$ and $\bar{T}$ are substituted for each other, and the remaining characteristics are taken into the diagram, too (Fig. 6).

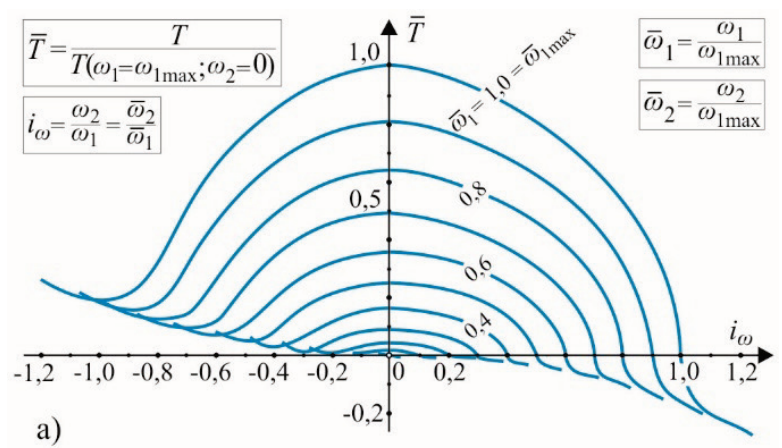

a)

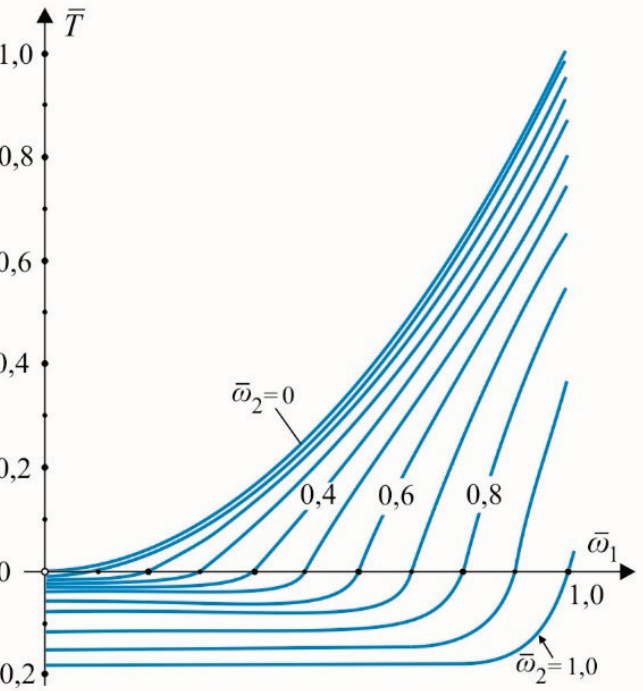

Figure 5 Steady state mechanical characteristics of $\mathrm{HC}$ represented in usual (a) and transformed forms (b)

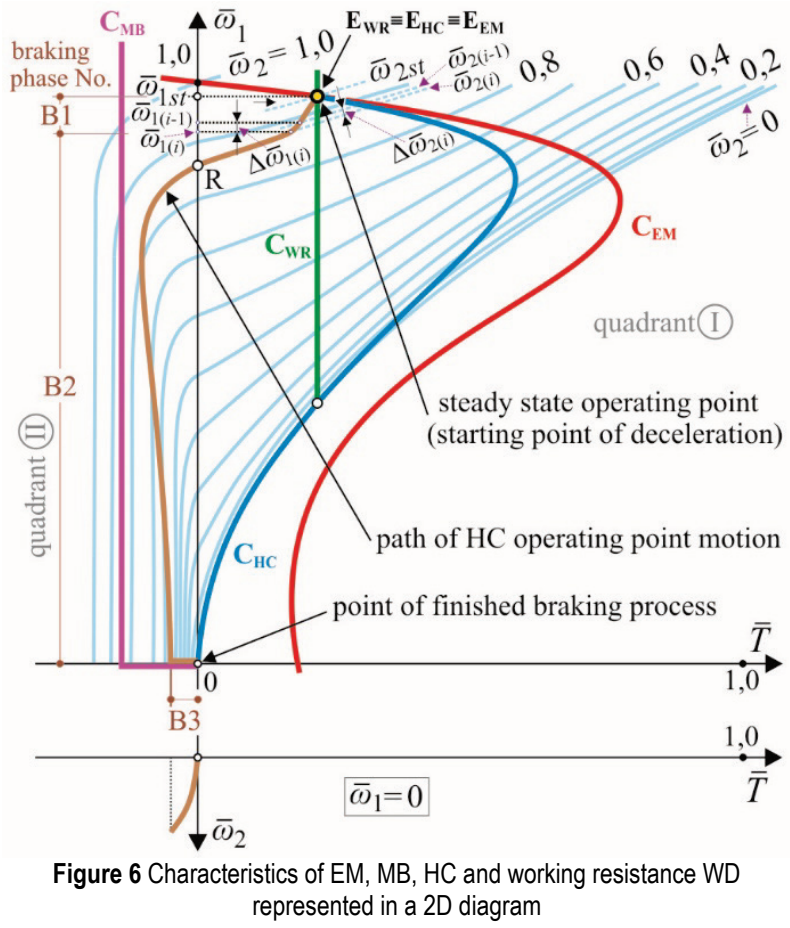

Further explanations concerning the determination of braking period duration are based on the diagram in Fig. 6 . 
In Fig. 6 the MB characteristic is in quadrant II. Its vertical part corresponds to the rotation of MB moving part, and horizontal part lying on the $\bar{T}$-axis to its standstill, while the part $J_{2}$ is still running (the added lower part of diagram enables the survey of $J_{2}$ motion in this phase).

\subsection{Braking Phase B1}

Motion of the EDS components during the braking phase B1 (see Fig. 2.b) is defined by the yoked equations:

$$
\begin{aligned}
& -\bar{T}_{H C}\left(\bar{\omega}_{1} ; \bar{\omega}_{2}\right)=J_{1} \cdot \frac{d \bar{\omega}_{1}}{d t}=J_{1} \cdot \dot{\bar{\omega}}_{1} \\
& \bar{T}_{H C}\left(\bar{\omega}_{1} ; \bar{\omega}_{2}\right)-\bar{T}_{W R}\left(\bar{\omega}_{2}\right)=J_{2} \cdot \frac{d \bar{\omega}_{2}}{d t}=J_{2} \cdot \dot{\bar{\omega}}_{2}
\end{aligned}
$$

By using the GM method for solving the previous equations, all the continuous functions are replaced with piecewise functions, and the rectangle method is applied. Calculation of the deceleration duration in the observed braking phase is carried out in $i$ steps (with $i=1,2,3, \ldots m$ ). Determination of the relevant values is in each step carried out iteratively (by the number of iterations $k=1,2,3, \ldots, j$ ), untill achieving the desired result accuracy $\varepsilon_{l}$. This iterative procedure is explained in [2], on an example of an EDS run-up. The accuracy $\varepsilon_{l}=0,03 \div 0,05$ is in the engineering practice considered to be quite satisfying for an approximate estimate of an EDS transient operating regime duration. In the further text, the calculation procedure in the step $i$ is outlined.

At the begining of a step $i$ values obtained for the step $i-1: \Delta \bar{\omega}_{1(i-1)}, \Delta \bar{\omega}_{2(i-1)}, \bar{T}_{H C(i-1)}$, and time $t_{(i-1)}$ are to be known.

The initial value of the step width $\Delta \bar{\omega}_{1(i)}$ can be arbitrary adopted. However, it is very desirable to narrow down the step width at the function sections with abrupt value changes, in aim to obtain better accuracy of the calculation.

Initial values of the $\mathrm{HC}$ torque and angular velocity of the turbine impeller before carrying out the first iteration of step $i$ are as follows:

$$
\begin{aligned}
& \bar{T}_{H C(i)}^{0}\left(\bar{\omega}_{1(i-1)}+\frac{\Delta \bar{\omega}_{1(i)}}{2} ; \bar{\omega}_{2(i-1)}\right) \\
& \Delta \bar{\omega}_{2(i)}^{0}=\frac{J_{1}}{J_{2}} \cdot\left(\frac{\bar{T}_{W R}}{\bar{T}_{H C(i)}^{0}}-1\right) \cdot \Delta \bar{\omega}_{1(i)}
\end{aligned}
$$

In the first step $(i=1)$ the initial values of angular velocities correspond to the steady state EDS operation: $\bar{\omega}_{1(i-1)}=\bar{\omega}_{1 s t}$ and $\bar{\omega}_{2(i-1)}=\bar{\omega}_{2 s t}$, and the initial torque value is $\bar{T}_{H C(i-1)}=\bar{T}_{W R}$, at the moment $t_{(i-1)}=0$.

In the $k$-th iteration the HC torque value is read off the 2D diagram (Fig. 6) as the value in the midpoint of step $i$ :

$$
\bar{T}_{H C(i)}^{k}\left(\bar{\omega}_{1(i-1)}+\frac{\Delta \bar{\omega}_{1(i)}}{2} ; \bar{\omega}_{2(i-1)}+\frac{\Delta \omega_{2(i)}^{k-1}}{2}\right)
$$

while the change of angular velocity of the turbine impeller is to be calculated according to the equation:

$\Delta \bar{\omega}_{2(i)}^{k}=\frac{J_{1}}{J_{2}} \cdot\left(\frac{\bar{T}_{W R}}{\bar{T}_{H C(i)}^{k}}-1\right) \cdot \Delta \bar{\omega}_{1(i)}$

Accuracy achieved in an iterative step $k$ is defined as:

$\varepsilon^{k}=\left|\frac{\Delta \bar{\omega}_{2(i)}^{k-1}-\Delta \bar{\omega}_{2(i)}^{k}}{\Delta \bar{\omega}_{2(i)}^{k-1}}\right| \leq \varepsilon_{l}$

Iterations are to be repeated to the step $k=j$, i.e. until the condition $\varepsilon^{k=j} \leq \varepsilon_{l}$ is fulfilled.

Time intervals corresponding to the step $i$ are to be defined separately for both parts of a regarded EDS model according to the relations:

$$
\begin{aligned}
\Delta t_{1(i)} & =\frac{J_{1} \cdot \Delta \bar{\omega}_{1(i)}}{-\bar{T}_{H C(i)}} \\
\Delta t_{2(i)} & =\frac{J_{2} \cdot \Delta \bar{\omega}_{2(i)}}{\bar{T}_{H C(i)}-\bar{T}_{W R}}
\end{aligned}
$$

It can be adopted that $\Delta t_{(i)} \approx 0,5 \cdot\left(\Delta t_{1(i)}+\Delta t_{2(i)}\right)$, giving the corresponding duration of deceleration in the step $i$ as: $t_{(i)} \approx t_{(i-1)}+\Delta t_{(i)}$. Values obtained in the step $i$ are initial values for the next step of calculation. In this phase, the steps are to be repeated to the step $i=m$, when the condition $t$ $(i=m) \geq t_{M B d}$ is fulfilled. Time interval $t_{M B d}$ is defined on the basis of brake catalogue data or according to the values recommended in literature.

\subsection{Braking Phase B2}

Final values obtained in the braking phase $\mathrm{B} 1$ and $t_{M B d}$ are the initial values for the calculations in the phase B2. Motion of the EDS components in the phase B2 is defined by the yoked Eqs. (2) and (7):

$$
-\bar{T}_{M B}\left(\bar{\omega}_{1}\right)-\bar{T}_{H C}\left(\bar{\omega}_{1} ; \bar{\omega}_{2}\right)=J_{1} \cdot \frac{d \bar{\omega}_{1}}{d t}=J_{1} \cdot \dot{\bar{\omega}}_{1}
$$

Further GM procedure is carried out by the analogous procedure used for the phase B1. In this case, the angular velocity of the turbine impeller is:

$$
\Delta \bar{\omega}_{2(i)}^{k}=\frac{J_{1}}{J_{2}} \cdot\left(\frac{\bar{T}_{W R}-\bar{T}_{H C(i)}^{k}}{\bar{T}_{M B}+\bar{T}_{H C(i)}^{k}}\right) \cdot \Delta \bar{\omega}_{1(i)}
$$

Time intervals corresponding to the step $i$ are to be defined according to Eqs. (6) and (9):

$$
\Delta t_{1(i)}=\frac{J_{1} \cdot \Delta \bar{\omega}_{1(i)}}{-\bar{T}_{M B}-\bar{T}_{H C(i)}}
$$

The braking phase B2 comes to an end at the moment of the pump impeller stopping $\left(\omega_{1}=0\right)$. In this phase, the $T_{H C}$ torque value in all probability changes the sign (point $\mathrm{R}$ in Fig. 6). Theoretically, it is possible that during the 
braking the turbine impeller comes to a standstill before the pump impeller (without change of a $T_{H C}$ value sign), but it is very unlikely in a case of a real belt conveyor drive structure, due to a MB installed on the pump impeller shaft between an EM and a HC.

\subsection{Braking Phase B3}

Final values obtained in the braking phase B2 are the initial values for the calculations in the phase B3. During this phase the $T_{M B}$ value varies with a change of $T_{H C}$ value, i.e. due to $\left|T_{M B}\right|=\left|T_{H C}\right|, T_{M B}$ value is no more constant. Part $J_{1}$ is already stopped, so, motion of the part $J_{2}$ is defined by Eq. (10), and time intervals corresponding to the step $i$ are defined by Eq. (6) and arbitrary chosen value of $\bar{\omega}_{2(i)}$.

$$
\bar{T}_{H C}\left(\bar{\omega}_{1}=0 ; \bar{\omega}_{2}\right)-\bar{T}_{W R}\left(\bar{\omega}_{2}\right)=J_{2} \cdot \frac{d \bar{\omega}_{2}}{d t}=J_{2} \cdot \dot{\bar{\omega}}_{2}
$$

The stopping time in the braking phase B3 is the total EDS braking period duration. In case that the initial time value in all braking phases is $=0$, the total braking time is defined by summing up the durations of all three phases.

In aim to survey the change of $T_{H C}$ value due to the change of $\omega_{2}$, it is necessary to add the diagram $\omega_{2}-T_{H C}$. (see the lower segment of diagram in Fig. 6). In fact, the added diagram is the projection of one part (of interest) of the 3D diagram in Fig. 4 onto the $\omega_{2}-T$ plane with $\omega_{1}=0$, and actually shows the characteristic of a hydrodynamic retarder. In this case, form of the $\omega_{2}-T$ curve corresponds to the curve form of a retarder without regulation (i.e. no adjusting the amount of filling), due to the adopted $\mathrm{HC}$ with constant fill.

Because of the considerable amount of mechanical energy that is during braking transformed into the thermal energy (heat), the $\mathrm{HC}$ has to be previously verified, and supplied with a cooling system for the HC working fluid.

\section{CONCLUSIONS}

The proposed method based on the joint 3D diagram containing the mechanical characteristics of relevant drive components (EM, HC, WD, and $\mathrm{MB}$ ) offers simultaneous visual survey of operation in all working regimes (steady state and transient: run-up, decelerating, and braking), and enables an easy understanding of their mutual dependence. The method in the presented form is not suitable in cases when some mechanical characteristic is varied with the additional controlling system, e.g. EM supply voltage or frequency, quantity of fluid in the $\mathrm{HC}$ working chamber, angle of $\mathrm{HC}$ impeller blades, etc. However, such systems are rarely used in usual belt conveyors due to additional expenses. Despite the mentioned limitations, the method enables simple defining of braking period duration based on catalogue data of EDS drive components. The method requests neither complex mathematical apparatus, nor a demanding software support for simulations, nor results of previous experiments. The obtained calculation results are of an approximate accuracy, but quite usable (and probably the only possible) at the stage of machine design, for defining the expected duration of the transient operating regimes. Simplifications used in the proposed method have no influence upon its general applicability. In a slightly expanded form, the method is applicable in the case of a hydrodynamic transformer, too.

The usage of the corresponding software extremely shortens the iterative procedure duration.

The method is of a notable educative importance, too.

\section{REFERENCES}

[1] Uzelac, D., Šostakov, R., \& Tašin, S. (1998). Starting of an electric motor drive with hydrodynamic coupling. FU Mech Eng, 1(5), 537-545.

[2] Šostakov, R., Uzelac, D., \& Brkljač, N. (2005). Methodology of surveying a driving mechanism operation with hydrodynamic coupling and calculation of speed-up duration (in Serbian). Tehnika-Mašinstvo, 54(3), 17-24.

[3] Šostakov, R., Zelić, A., \& Poznić, A. (2015). Survey of a machine driving system operation in $2 \mathrm{D}$ and $3 \mathrm{D}$ diagrams torque vs. angular velocity. In László Gogolák, Igor Fürstner (Eds.), Proceedings of Mechedu 2015, Subotica: Subotica tech - College of Applied Sciences, 168-171.

[4] Bogdanović, B., Spasić, Ž., \& Bogdanović-Jovanović, J. (2005). Calculation of the starting regime of the power transmission system with a hydrodynamic coupling and a driving motor. FU Mech Eng, 3(1), 59-68.

[5] Höller, H. (1996). Hydrodynamische Kupplungen im Antrieb von Gurtförderern. Fördern und Heben, 46(5), 396-399.

[6] Fries, J. \& Hapla, T. (2018). Influence affecting the lifetime of belt conveyor's drive drums. Tehnički vjesnik, 25(Suppl. 1), 714. https://doi.org/10.17559/TV-20140411120832

[7] Kulinowski, P. (2004). Dynamic start-up calculations for belt conveyors with measured torque curves of fluid couplings. In Hardygóra, M., Paszkowska, G., \& Sikora, M. (Eds.), Proceedings of Mine Planning and Equipment Selection 2004/Wroclaw, London: CRC Press, 443-448

[8] He, D., Pang, Y., \& Lodewijks, G. (2016). Speed control of belt conveyors during transient operation. Powder Technology, 301, 622-631. http://dx.doi.org/10.1016/j.powtec.2016.07.004

[9] Khatir, T., Bouchetara, M., \& Boutchicha, D. (2014). Dynamics study of the operating behavior of hydrodynamic coupling by experimental and numerical simulation. Mechanika, 20(4), 395-401. http://dx.doi.org/10.5755/j01.mech.20.4.6297

[10] Behrens, H., Jaschke, P., Steinhausen, J., \& Waller, H. (2000). Modelling of Technical Systems: Application to Hydrodynamic Torque Converters and Couplings. Math. Comput. Model. Dyn. Syst, 6(3), 223-250. DOI: 10.1076/1387-3954(200009)6:3;1-I;FT223

[11] Masaki, M. S., Zhang L., \& Xia, X. (2018). A design approach for multiple drive belt conveyors minimizing life cycle costs. Journal of Cleaner Production, 201, 526-541. https://doi.org/10.1016/j.jclepro.2018.08.040

[12] Bebic, M. \& Ristic, L. (2018). Speed Controlled Belt Conveyors: Drives and Mechanical Considerations. Adv. Electr. Comp. Eng., 18(1), 51-60. https://doi.org/10.4316/AECE.2018.01007

Contact information:

Atila ZELIĆ, PhD

Rastislav ŠOSTAKOV, PhD

Dragan ŽIVANIĆ, PhD (Corresponding author)

Faculty of Technical Sciences, University of Novi Sad,

Trg Dositeja Obradovića 6, 21000 Novi Sad, Republic of Serbia

zelic@uns.ac.rs

sostakov@uns.ac.rs

zivanic@uns.ac.rs 